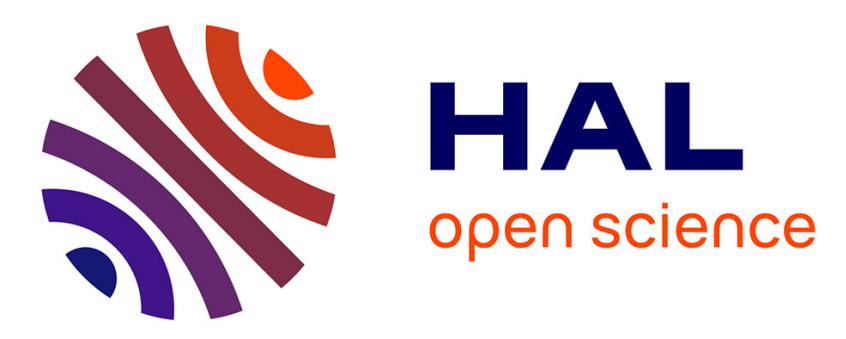

\title{
Brillouin scattering and three-body forces in argon at high pressures
}

\author{
M Grimsditch, ' P Loubeyre, A. Polian
}

\section{To cite this version:}

M Grimsditch, ' P Loubeyre, A. Polian. Brillouin scattering and three-body forces in argon at high pressures. Physical Review B: Condensed Matter (1978-1997), 1986. hal-03059417

\section{HAL Id: hal-03059417 \\ https://hal.science/hal-03059417}

Submitted on 12 Dec 2020

HAL is a multi-disciplinary open access archive for the deposit and dissemination of scientific research documents, whether they are published or not. The documents may come from teaching and research institutions in France or abroad, or from public or private research centers.
L'archive ouverte pluridisciplinaire HAL, est destinée au dépôt et à la diffusion de documents scientifiques de niveau recherche, publiés ou non, émanant des établissements d'enseignement et de recherche français ou étrangers, des laboratoires publics ou privés. 


\title{
Brillouin scattering and three-body forces in argon at high pressures
}

\author{
M. Grimsditch, ${ }^{*}$ P. Loubeyre, and A. Polian \\ Laboratoire de Physique des Milieux Très Condensés, Université Pierre et Marie Curie (Paris VI), \\ Tour 13, Quatrième Etage, 4 place Jussieu, F-75230 Paris Cédex 05, France
}

(Received 21 January 1986)

\begin{abstract}
Brillouin scattering was measured at room temperature in liquid and solid argon in a diamondanvil cell at pressures up to $33 \mathrm{GPa}$. In the fluid, our results agree well with those obtained by ultrasonic methods. In the solid, the elastic anisotropy and the use of the experimental equation of state allowed us to determine limiting values for all the elastic constants as a function of pressure. We also performed self-consistent phonon calculations using various pair potentials proposed for argon. From the comparison between calculation and experiment it can be stated that in dense solid argon there is no way to reproduce the experimental results with any pair potential and that manybody exchange potentials have to be incorporated for an accurate description.
\end{abstract}

\section{INTRODUCTION}

The description of the properties of condensed matter in terms of the electronic properties of its constituent atoms has been one of the main objectives of modern solid-state physics. In most cases, however, complete $a b$ initio calculations are not possible due to their complexity. A notable exception to this general rule is the rare-gas solids. These systems, because of their simple electronic configurations, which allow the atoms to be treated as spherically symmetric and also which almost always crystallize in a cubic structure, are ideal testing grounds for calculations. From a theoretical standpoint, the concept of pair interactions has brought major advances in this field where pair potentials for $\mathrm{He}, \mathrm{Ne}$, and Ar have been accurately determined. ${ }^{1}$ Until recently, essentially all the experimentally determined properties of condensed rare gases could be calculated using these potentials and subsequently extrapolated to domains where no experimental results existed. In this respect one could say that "theory was ahead of experiments."

The amount of experimental results accumulated on the rare-gas solids is considerable. ${ }^{2}$ The properties of these systems are consequently well known as a function of temperature and pressure up to $2 \mathrm{GPa}$. Above this pressure, however, almost no experimental results existed. One of the main reasons for the lack of experimental results at high pressures, which can now routinely be achieved with the use of diamond-anvil cells (DAC), is that these solids almost always crystallize in structures that are neither Raman nor ir active, thereby excluding two of the most powerful techniques used in DAC studies. $^{3}$ Brillouin scattering, which only recently has become applicable in conjunction with a $\mathrm{DAC},{ }^{4-7}$ is then one of the few optical techniques available to study rare-gas solids at very high pressures.

In Sec. II we briefly review the technique of Brillouin scattering, describe the experimental apparatus used, and also describe the rather simple method used to load the cell. Experimental results are presented in Sec. III. In Sec. IV the methods used to calculate the properties of solid argon will be briefly described and the results obtained with various known potentials presented. Section $\mathrm{V}$ contains the comparison between experiment and theory. Our conclusions are summarized in Sec. VI.

\section{BACKGROUND AND EXPERIMENTAL SETUP}

The Brillouin scattering experiments were carried out using a five-pass Fabry-Perot interferometer and $200 \mathrm{~mW}$ of 514.5-nm radiation from a single-moded Ar laser. The diamond-anvil cell was of the Block-Piermarini ${ }^{8}$ type and hence all experiments were restricted to the backscattering geometry. In this scattering geometry the shift (in $\mathrm{cm}^{-1}$ ) of the scattered radiation is given by

$$
\Delta \sigma=2 n v / \lambda c,
$$

where $n$ is the refractive index of the medium, $\lambda$ the wavelength of the incident radiation, $c$ the velocity of light, and $v$ the velocity of sound in the medium. Furthermore, since in this scattering geometry coupling of the light occurs almost exclusively to the longitudinal phonons, ${ }^{9}$ only one peak (Stokes and anti-Stokes) originating from the sample is observed. The free spectral range of the interferometer was chosen so as to hide the very intense Brillouin scattering from the diamond anvils ${ }^{10}$ in the unshifted laser line.

Loading is done with the setup shown in Fig. 1; the shaded areas are added to the diamond-anvil cell to form a cap above the fixed diamond. The volume under the cap can be purged and then held at a pressure slightly above $1 \mathrm{~atm}(0.12 \mathrm{MPa})$. Gas leakage around the piston is small enough so as to pose no problem. The whole assembly is then placed in liquid $\mathrm{N}_{2}$, leaving the lucite cover above the liquid. The hole in the gasket can then be observed under a microscope until the liquid is seen to condense into the hole; at this point, the cell can be closed and the system removed from the liquid $\mathrm{N}_{2}$. This method is obviously applicable to any gas that liquifies above 77 $\mathrm{K}$ (i.e., not $\mathrm{He}, \mathrm{Ne}$, or $\mathrm{H}_{2}$ ) and has been used by us to load $\mathrm{Kr}$ and $\mathrm{CH}_{4}$. Furthermore, this method probably can be used with many of the standard diamond-anvil cells. We 


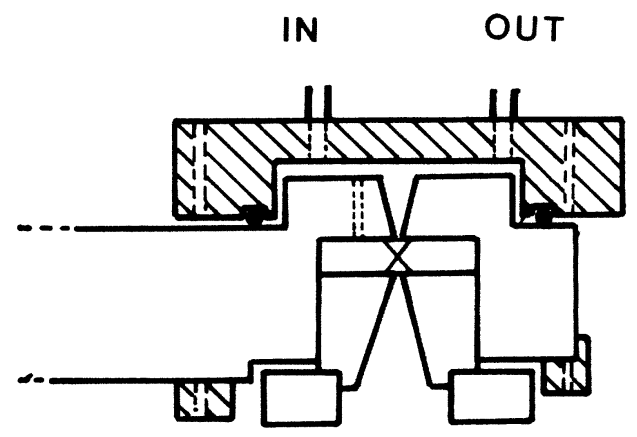

FIG. 1. Schematic diagram of the system used to load the cell with argon. The cross-hatched areas are the additions to the standard cell. The upper portion, made of lucite, is screwed to the two metal bars below. An $O$-ring seals the cell and the lucite.

may also mention that an extension of this method, in which liquid $\mathrm{He}$ at a pressure of 2 bar was used as a coolant, has allowed a cell to be filled with $\mathrm{He}$.

Once $\mathrm{Ar}$ is in the cell is it possible to obtain a single crystal in the same manner as previously described for $\mathrm{H}_{2} \mathrm{O}{ }^{11}$ A single argon crystal in equilibrium with the liquid is observed to be almost circular and shows no sign of faceting; hence, no information is available as to its orientation.

The pressure was measured using the nonlinear ruby fluorescence scale, ${ }^{12}$ viz.,

$$
P(\mathrm{GPa})=380.8\left[\left(\frac{\lambda}{\lambda_{0}}\right)^{5}-1\right],
$$

where $\lambda$ and $\lambda_{0}$ are the positions of the ruby fluorescence peaks at pressures $P$ and zero, respectively.

\section{RESULTS AND DISCUSSION}

In Fig. 2 we present the measured frequency shift as a function of pressure. Using Eq. (1) we obtain values for $n v$ which are indicated on the right-hand side of Fig. 2. Furthermore, since these results may be useful to test future theoretical calculations, we also present them in tabular form in Table I.

The results in Fig. 2 clearly show the fluid-solid transition at $1.35 \mathrm{GPa}$ which is in good agreement with previous determinations of the melting curve. ${ }^{13}$ Below the melting point our experimental results can be seen to be very reproducible and our major source of error in this region is in the pressure determination. Above $1.4 \mathrm{GPa}$ the spread in the experimental results is greater than the experimental error and can be traced to the elastic anisotropy of argon. This point will be discussed in greater detail in the next section.

In order to evaluate the velocities and the elastic constants of argon from our experimental results, it is necessary [see Eq. (1)] to know the refractive index as a function of pressure. Since the refractive index of argon has not been measured at high pressures we use the results obtained from calculations. In Ref. 14, the refractive index

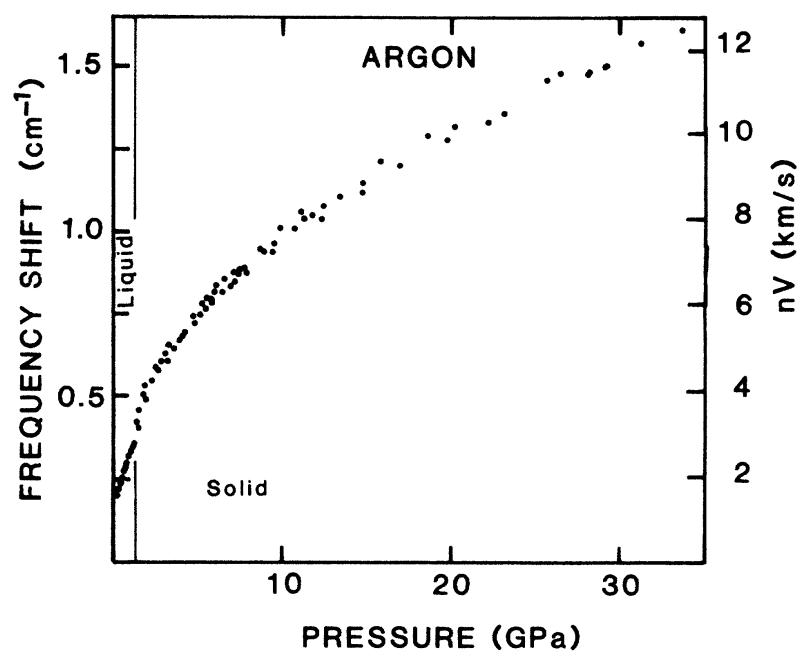

FIG. 2. Brillouin frequency shift from argon as a function of pressure (left-hand scale) and the corresponding values of the product of the refractive index times the sound velocity (righthand scale).

of xenon was calculated accurately using a one-electron model for the dielectric constant. The use of the corresponding-states law led to the following equations: $:^{14}$

$$
3 \frac{n^{2}-1}{n^{2}+2}=\left(\frac{5.4}{E_{b}^{2}-E^{2}}+3.125 \times 10^{-4}\right),
$$

where

$$
E_{b}=19.23+0.381|0.9107 \rho-1|^{1.8} .
$$

$E$ is the photon energy at which the refractive index is calculated; $E$ and $E_{b}$ are in $\mathrm{eV}$ and $\rho$ in $\mathrm{g} / \mathrm{cm}^{3}$. Preliminary measurements up to $15 \mathrm{GPa}$ (Ref. 15) confirm the extrapolation.

We now analyze separately, in the following, the regions of fluid and solid.

(i) Fluid: In this region the density is well known and in Table I we list the densities obtained from Ref. 13 at the pressures of our measurements. The refractive index measured in the liquid ${ }^{16}$ [with which the calculations based on Eqs. (3) and (4) agree well] is also listed in Table I. With these values of $n$, the sound velocity $v$ and the adiabatic bulk modulus $B_{s}$, defined by

$$
B_{s}=\rho v^{2},
$$

can be calculated and are listed in Table I. In Fig. 3 we plot our values of $B_{s}$ (open circles), which can be seen to be in good agreement with those of Ref. 13 (dots).

(ii) Solids: In the solid, the density has been determined by $x$ rays up to $77 \mathrm{GPa}^{17-19}$ and is plotted in Fig. 4 (dots). We have fitted these results to a polynomial of the form

$$
P=\sum_{i} A_{i} \rho^{i} .
$$

for different values of $i$ and find that for $i>3$ the sum of the "square errors" no longer diminishes appreciably. 
Hence, we have taken $i=3$ and obtain $A_{0}=12.65$, $A_{1}=-11.43, A_{2}=1.5$, and $A_{3}=0.68$ when $P$ is in $\mathrm{GPa}$ and $\rho$ in $\mathrm{g} / \mathrm{cm}^{3}$. The solid curve in Fig. 4 represents this fit and it can be seen that it describes the experimental results extremely well. The isothermal bulk modulus $B_{T}$ (which apart from a small region near melting can be tak- en to be equal to the adiabatic one) can be calculated from Eq. (6) using

$$
B=B_{s}=B_{T}=\rho \frac{d P}{d \rho} .
$$

The values of $B$ thus obtained turned out to be very sensi-

TABLE I. Experimental results for Brillouin scattering at various pressures. For each pressure is given the Brillouin shift $\Delta \sigma$ in $\mathrm{cm}^{-1}, n v$ deduced through Eq. (1), the density taken from Refs. 17-19, the refractive index $n$ [measured in the liquid (Ref. 16) and calculated in the solid using Eqs. (4) and (5)], and the effective elastic constant $C=\rho_{v}^{2}$.

\begin{tabular}{|c|c|c|c|c|c|c|c|c|c|c|c|}
\hline$P$ & $\Delta \sigma$ & $n v$ & $\rho$ & $n$ & $C$ & $P$ & $\Delta \sigma$ & $n v$ & $\rho$ & $n$ & $C$ \\
\hline 0.11 & 0.161 & 1.24 & 1.030 & 1.168 & 1.16 & 4.87 & 0.749 & 5.78 & 2.578 & 1.421 & 42.67 \\
\hline 0.13 & 0.153 & 1.18 & 1.090 & 1.178 & 1.09 & $\begin{array}{l}4.01 \\
4.92\end{array}$ & 0.724 & 5.59 & 2.583 & 1.421 & 39.95 \\
\hline 0.27 & 0.199 & 1.54 & 1.325 & 1.218 & 2.12 & 5.17 & 0.749 & 5.78 & 2.608 & 1.425 & 42.90 \\
\hline 0.28 & 0.196 & 1.51 & 1.340 & 1.220 & 2.05 & 5.35 & 0.780 & 6.02 & 2.625 & 1.428 & 46.67 \\
\hline 0.35 & 0.219 & 1.69 & 1.420 & 1.234 & 2.66 & 5.56 & 0.765 & 5.90 & 2.644 & 1.431 & 44.98 \\
\hline 0.38 & 0.223 & 1.72 & 1.445 & 1.238 & 2.79 & $\begin{array}{l}5.50 \\
5.56\end{array}$ & 0.803 & 6.20 & 2.644 & 1.431 & 49.67 \\
\hline 0.38 & 0.225 & 1.74 & 1.445 & 1.238 & 2.85 & 5.74 & 0.812 & 6.27 & 2.661 & 1.433 & 50.94 \\
\hline 0.39 & 0.225 & 1.74 & 1.450 & 1.239 & 2.86 & 5.82 & 0.796 & 6.14 & 2.668 & 1.434 & 48.91 \\
\hline 0.47 & 0.240 & 1.85 & 1.515 & 1.250 & 3.32 & 6.00 & 0.820 & 6.33 & 2.684 & 1.436 & 52.13 \\
\hline 0.48 & 0.239 & 1.84 & 1.525 & 1.251 & 3.30 & 6.03 & 0.834 & 6.44 & 2.687 & 1.437 & 53.98 \\
\hline 0.55 & 0.251 & 1.94 & 1.570 & 1.259 & 3.73 & 6.45 & 0.814 & 6.28 & 2.723 & 1.442 & 51.65 \\
\hline 0.55 & 0.254 & 1.96 & 1.570 & 1.259 & 3.81 & 6.53 & 0.854 & 6.59 & 2.730 & 1.443 & 56.94 \\
\hline 0.65 & 0.270 & 2.08 & 1.630 & 1.269 & 4.38 & 6.63 & 0.858 & 6.62 & 2.738 & 1.444 & 57.53 \\
\hline 0.66 & 0.273 & 2.11 & 1.635 & 1.270 & 4.51 & 7.02 & 0.837 & 6.46 & 2.769 & 1.449 & 55.07 \\
\hline 0.76 & 0.287 & 2.21 & 1.700 & 1.281 & 5.06 & 7.15 & 0.877 & 6.77 & 2.779 & 1.450 & 60.58 \\
\hline 0.82 & 0.293 & 2.26 & 1.725 & 1.285 & 5.34 & 7.16 & 0.850 & 6.56 & 2.780 & 1.450 & 56.89 \\
\hline 0.91 & 0.309 & 2.38 & 1.770 & 1.288 & 6.05 & 7.41 & 0.886 & 6.84 & 2.800 & 1.453 & 62.05 \\
\hline 0.99 & 0.319 & 2.46 & 1.800 & 1.294 & 6.50 & $\begin{array}{l}1.41 \\
7.50\end{array}$ & 0.885 & 6.83 & 2.806 & 1.454 & 61.93 \\
\hline 1.01 & 0.320 & 2.47 & 1.810 & 1.299 & 6.54 & 7.91 & 0.873 & 6.74 & 2.837 & 1.458 & 60.61 \\
\hline 1.02 & 0.332 & 2.56 & 1.815 & 1.300 & 7.04 & 7.91 & 0.893 & 6.89 & 2.837 & 1.458 & 63.33 \\
\hline 1.12 & 0.337 & 2.60 & 1.850 & 1.306 & 7.33 & 8.68 & 0.944 & 7.29 & 2.892 & 1.466 & 71.52 \\
\hline 1.24 & 0.350 & 2.70 & 1.890 & 1.312 & 8.00 & 8.89 & 0.942 & 7.27 & 2.906 & 1.468 & 71.29 \\
\hline 1.36 & 0.354 & 2.73 & 1.920 & 1.314 & 8.29 & 9.47 & 0.939 & 7.25 & 2.945 & 1.473 & 71.33 \\
\hline 1.31 & 0.427 & 3.30 & 2.017 & 1.333 & 12.36 & 9.55 & 0.967 & 7.46 & 2.951 & 1.474 & 75.59 \\
\hline 1.47 & 0.421 & 3.25 & 2.066 & 1.341 & 12.14 & 9.96 & 1.008 & 7.78 & 2.977 & 1.477 & 82.54 \\
\hline 1.47 & 0.459 & 3.54 & 2.066 & 1.341 & 14.40 & 10.81 & 1.009 & 7.79 & 3.030 & 1.485 & 83.42 \\
\hline 1.53 & 0.403 & 3.11 & 2.083 & 1.344 & 11.16 & 11.24 & 1.059 & 8.17 & 3.056 & 1.488 & 92.12 \\
\hline 1.81 & 0.502 & 3.87 & 2.151 & 1.355 & 17.56 & 11.34 & 1.038 & 8.01 & 3.062 & 1.489 & 88.63 \\
\hline 1.93 & 0.535 & 4.13 & 2.177 & 1.359 & 20.11 & 11.78 & 1.053 & 8.13 & 3.087 & 1.492 & 91.64 \\
\hline 1.98 & 0.489 & 3.77 & 2.187 & 1.360 & 16.80 & 12.36 & 1.042 & 8.04 & 3.120 & 1.496 & 90.07 \\
\hline 2.26 & 0.544 & 4.20 & 2.240 & 1.369 & 21.09 & 12.47 & 1.080 & 8.33 & 3.126 & 1.497 & 96.77 \\
\hline 2.53 & 0.591 & 4.56 & 2.286 & 1.376 & 25.10 & 13.45 & 1.107 & 8.54 & 3.180 & 1.504 & 102.5 \\
\hline 2.69 & 0.587 & 4.53 & 2.312 & 1.380 & 24.91 & 14.71 & 1.120 & 8.64 & 3.244 & 1.512 & 105.9 \\
\hline 3.00 & 0.603 & 4.65 & 2.358 & 1.387 & 26.49 & 14.79 & 1.147 & 8.85 & 3.249 & 1.513 & 111.2 \\
\hline 3.05 & 0.643 & 4.96 & 2.364 & 1.388 & 30.18 & 15.91 & 1.215 & 9.38 & 3.304 & 1.519 & 125.9 \\
\hline 3.07 & 0.614 & 4.74 & 2.367 & 1.389 & 27.58 & 16.87 & 1.204 & 9.29 & 3.348 & 1.525 & 124.3 \\
\hline 3.16 & 0.610 & 4.71 & 2.380 & 1.391 & 27.30 & 18.59 & 1.293 & 9.98 & 3.426 & 1.534 & 145.0 \\
\hline 3.16 & 0.629 & 4.85 & 2.380 & 1.391 & 28.95 & 19.76 & 1.282 & 9.89 & 3.476 & 1.539 & 143.5 \\
\hline 3.25 & 0.646 & 4.99 & 2.392 & 1.393 & 30.72 & 20.23 & 1.321 & 10.19 & 3.496 & 1.542 & 152.7 \\
\hline 3.30 & 0.603 & 4.65 & 2.399 & 1.393 & 26.71 & 22.19 & 1.334 & 10.30 & 3.574 & 1.550 & 157.8 \\
\hline 3.30 & 0.655 & 5.05 & 2.399 & 1.393 & 31.50 & 23.19 & 1.365 & 10.53 & 3.613 & 1.554 & 165.8 \\
\hline 3.58 & 0.645 & 4.98 & 2.434 & 1.399 & 30.84 & 25.65 & 1.466 & 11.31 & 3.704 & 1.564 & 193.8 \\
\hline 3.87 & 0.678 & 5.23 & 2.470 & 1.404 & 34.25 & 26.40 & 1.483 & 11.45 & 3.731 & 1.566 & 199.4 \\
\hline 3.96 & 0.672 & 5.19 & 2.480 & 1.406 & 33.79 & 28.11 & 1.473 & 11.37 & 3.790 & 1.572 & 198.2 \\
\hline 4.08 & 0.678 & 5.23 & 2.494 & 1.408 & 34.41 & 28.22 & 1.489 & 11.49 & 3.794 & 1.573 & 202.6 \\
\hline 4.08 & 0.687 & 5.30 & 2.494 & 1.408 & 35.33 & 29.16 & 1.503 & 11.60 & 3.826 & 1.576 & 207.4 \\
\hline 4.35 & 0.697 & 5.38 & 2.524 & 1.413 & 36.61 & 29.19 & 1.506 & 11.62 & 3.826 & 1.576 & 208.1 \\
\hline 4.78 & 0.725 & 5.60 & 2.569 & 1.419 & 39.99 & 31.21 & 1.572 & 12.13 & 3.892 & 1.582 & 229.0 \\
\hline 4.79 & 0.745 & 5.75 & 2.570 & 1.420 & 42.17 & 33.58 & 1.612 & 12.44 & 3.936 & 1.585 & 242.3 \\
\hline
\end{tabular}




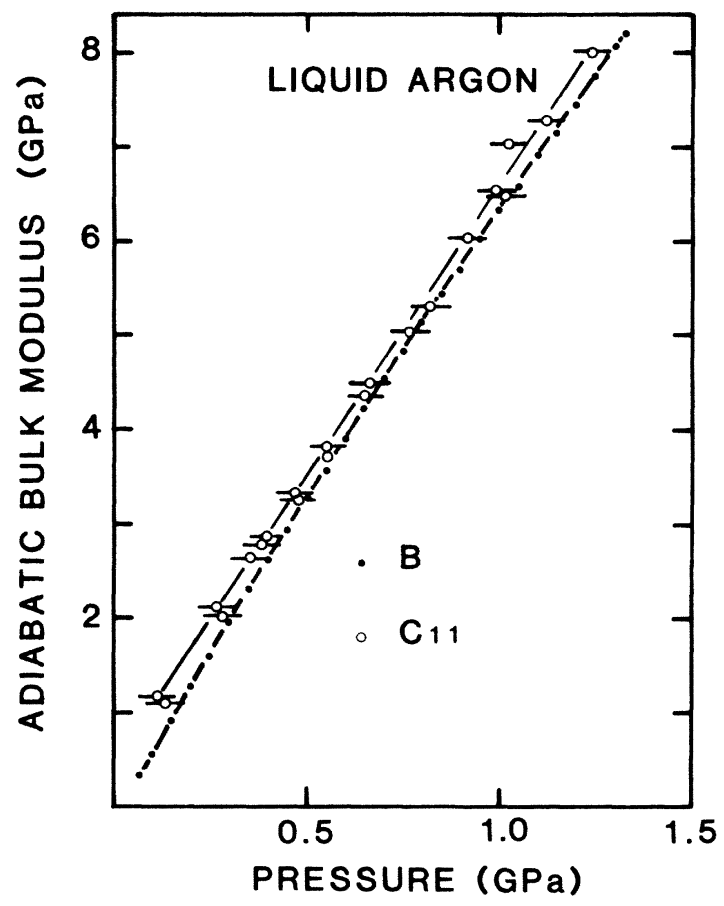

FIG. 3. Adiabatic bulk modulus of liquid argon versus pressure. Open circles are our results and the dots are from Ref. 13.

tive to the fit at low densities. From the different fits we estimate the error in $B$ to be $15 \%$ around $2 \mathrm{GPa}, 2 \%$ at 4 $\mathrm{GPa}$, and $1 \%$ at higher pressures ( $<60 \mathrm{GPa}$ ).

As for the liquid, the densities from Eq. (6) allow the refractive index, the velocity, and an effective elastic constant, defined by

$$
C=\rho v^{2},
$$

to be calculated. These are given in Table I. The values for $C$ are plotted in Fig. 5 as a function of pressure. The spread in experimental points is due to elastic anisotropy of the crystal, as was ascertained by repetitive cycling of the sample from liquid to $3 \mathrm{GPa}$ so that a new single crystal was produced each time. In the cases where no precautions were taken to produced a single crystal, the Brillouin peaks were usually considerably broadened.

From the elastic constants determined at low temperatures $^{20}$ and from theoretical calculations, it is known that a minimum in the longitudinal sound velocity exists along the $\langle 100\rangle$ directions and is given by

$$
v^{2}=C_{11} / \rho
$$

and a maximum along the $\langle 111\rangle$ directions is given by

$$
v^{2}=C^{*} / \rho
$$

where

$$
C^{*}=\frac{1}{3}\left(C_{11}+2 C_{12}+4 C_{44}\right) \text {. }
$$

If the orientations of the crystals formed in the cell were perfectly random we could interpret our results as a band, given by the dashed curve in Fig. 5, extending from

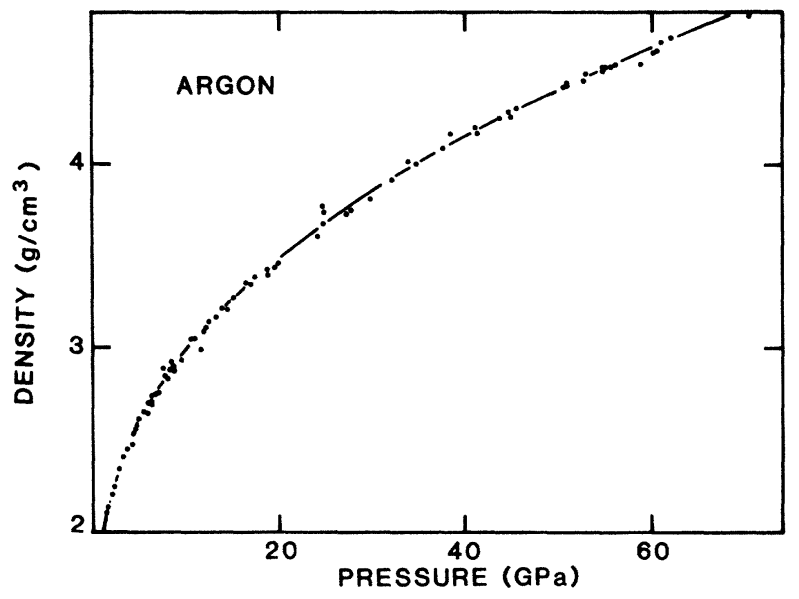

FIG. 4. Density of solid argon versus pressure. Dots: Refs. 17-19. Solid line: our fit to the data.

$C_{11}$ to $C^{*}$. However, due to the problem of sample reorientation in the cell (to be discussed in greater detail in a later paragraph), we can only interpret our results as a correct value for $C^{*}$ and only as an upper limit for $C_{11}$. If we combine our results with those of the bulk modulus given by Eqs. (6) and (7), using the fact that for a cubic crystal

$$
B=\frac{1}{3}\left(C_{11}+2 C_{12}\right) \text {, }
$$

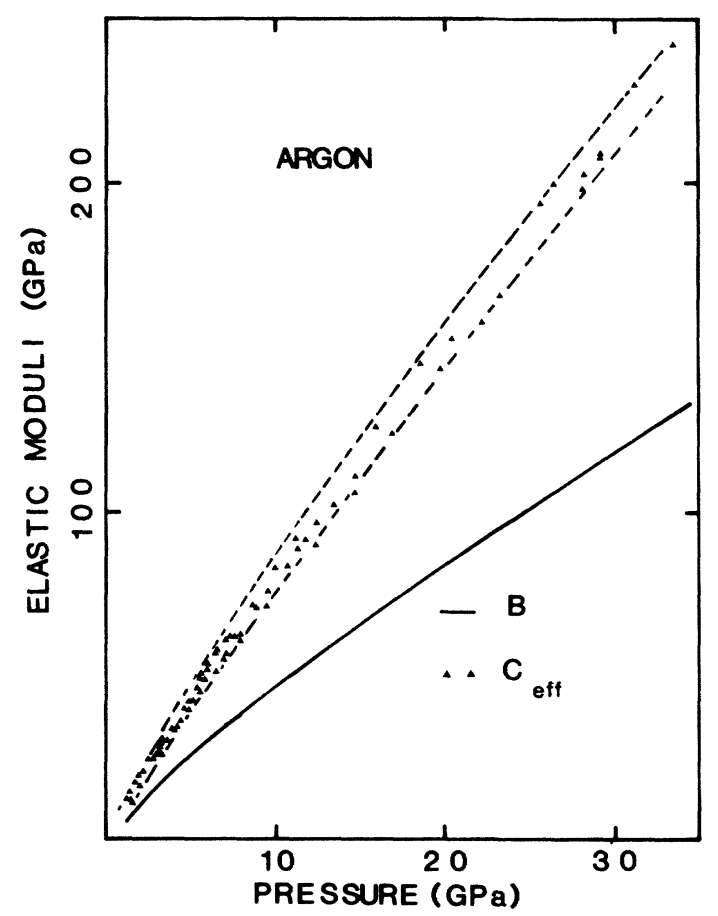

FIG. 5. Elastic moduli of solid argon versus pressure. Triangles: effective longitudinal moduli (defined in text) measured by Brillouin scattering; the two dashed lines represent the envelope curves we used to determine $C_{11}$ and $C^{*}$ (see text). Solid line: bulk modulus determined by $\mathrm{x}$-ray measurements (Refs. 17-19). 
TABLE II. Elastic constants of solid argon versus pressure. (All the quantities are in GPa.)

\begin{tabular}{rccccc}
\hline \hline$P$ & $B$ & $C^{*}$ & $C_{11}{ }^{\mathrm{a}}$ & $C_{44}$ & $C_{12}{ }^{\mathrm{b}}$ \\
\hline 2 & $11 \pm 1$ & $21 \pm 1$ & $16 \pm 1$ & $7.5 \pm 1.5$ & $8.4 \pm 2.0$ \\
4 & $21.6 \pm 0.5$ & $37 \pm 1$ & $32 \pm 1$ & $11.6 \pm 1.1$ & $16.3 \pm 1.3$ \\
6 & $30.6 \pm 0.5$ & $54 \pm 1$ & $48 \pm 1$ & $17.6 \pm 1.1$ & $21.9 \pm 1.3$ \\
10 & $46.9 \pm 0.5$ & $83 \pm 1$ & $74 \pm 1$ & $27.1 \pm 1.1$ & $33.3 \pm 1.3$ \\
15 & $65.6 \pm 0.6$ & $120 \pm 2$ & $109 \pm 2$ & $41 \pm 2$ & $44 \pm 2$ \\
20 & $83.5 \pm 0.8$ & $155 \pm 2$ & $142 \pm 2$ & $54 \pm 2$ & $54 \pm 2$ \\
25 & $101 \pm 1.0$ & $191 \pm 2$ & $178 \pm 2$ & $68 \pm 2$ & $63 \pm 3$ \\
30 & $118 \pm 1.0$ & $225 \pm 2$ & $211 \pm 2$ & $80 \pm 2$ & $71 \pm 3$ \\
\hline \hline
\end{tabular}

${ }^{a}$ For the upper bound.

${ }^{b}$ For the lower bound.

we can obtain a complete set of elastic constants, or at least upper and lower bounds for them as a function of pressure. These are given in Table II.

The phenomenon of crystal reorientation has been reported in Ref. 21. There, it was found that upon increasing pressure the sample could change its orientation so that certain preferential directions would lie along the cell axis. This effect, as observed in our Brillouin scattering measurements, is shown for a particular run in Fig. 6. Starting with a single crystal at $1.4 \mathrm{GPa}$, a single peak was observed up to $5.57 \mathrm{GPa}$ when two peaks were observed simultaneously. At higher pressures the lower peak slowly disappeared. On decreasing the pressure, the peaks became broader, consistent with the presence of a mixture of orientations. The increase observed at 5.57 $\mathrm{GPa}$ is consistent with a reorientation along a $\langle 111\rangle$ direction, as reported in Ref. 21. We have also observed the converse effect, viz., that on decreasing pressure, a sudden drop occurs in the position of the Brillouin peak. This could indicate a reorientation of the crystal along its softest direction, i.e., $\langle 100\rangle$, in which case the values of $C_{11}$ and $C_{12}$ given in Table II would be correct. However, until this assumption can be confirmed by $x$-ray measurements we will treat the values given for $C_{11}$ and $C_{12}$ as upper and lower bounds, respectively.

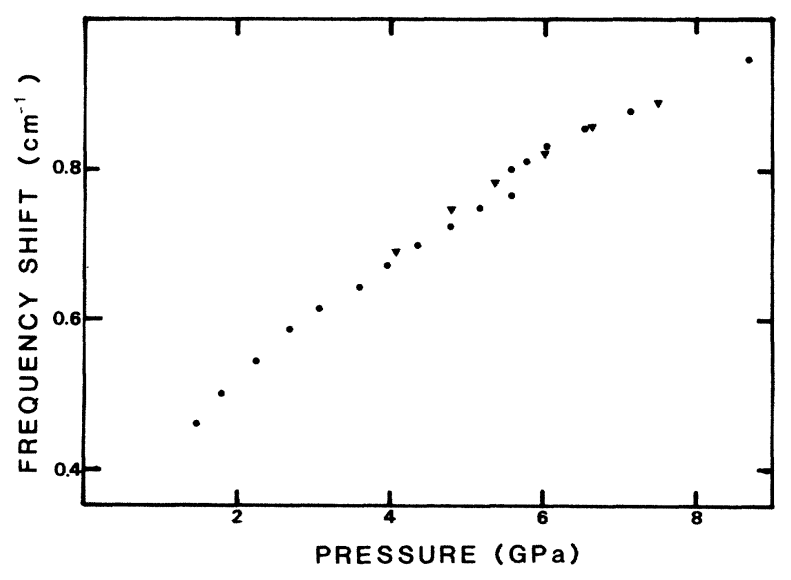

FIG. 6. Frequency shift versus pressure showing the effect of crystal reorientation. Dots: pressure increases. Triangles: pressure decreases.
In Fig. 7 we show the logarithm of the peak intensity, normalized to laser power and accumulation time, of the Brillouin lines of argon as a function of pressure. Using expressions for the intensity of Brillouin scattering (Ref. 22), we can expect

$$
I \propto \frac{n^{10}\left(p_{i j}\right)^{2} d}{\rho \Delta \sigma^{2}},
$$

where $d$ is the distance between the diamond anvils and $p_{i j}$ is an elasto-optic constant. Since in this expression all term except $p_{i j}$ can be evaluated, we conclude that $p_{i j}$ diminishes by a factor of $3.5 \pm 2$ when the pressure is increased from 2 to $8 \mathrm{GPa}$, where the error comes mainly from the measured intensities and the estimate of $d$.

As a final point we mention the use of argon as a pressure transmitting medium; it has sometimes been assumed that because of its "soft" nature it would be a good substance for that purpose. In our experiments we found that in spite of maintaining a relatively narrow linewidth (12 $\mathrm{cm}^{-1}$ ) up to $35 \mathrm{GPa}$, the equivalent pressure difference between two rubies at opposite ends of the hole in the gasket was as large as $1.5 \mathrm{GPa}$. Consequently, even though it certainly is better than the conventional methanol-ethanol mixture, it is still not a perfect hydrostatic pressure medium.

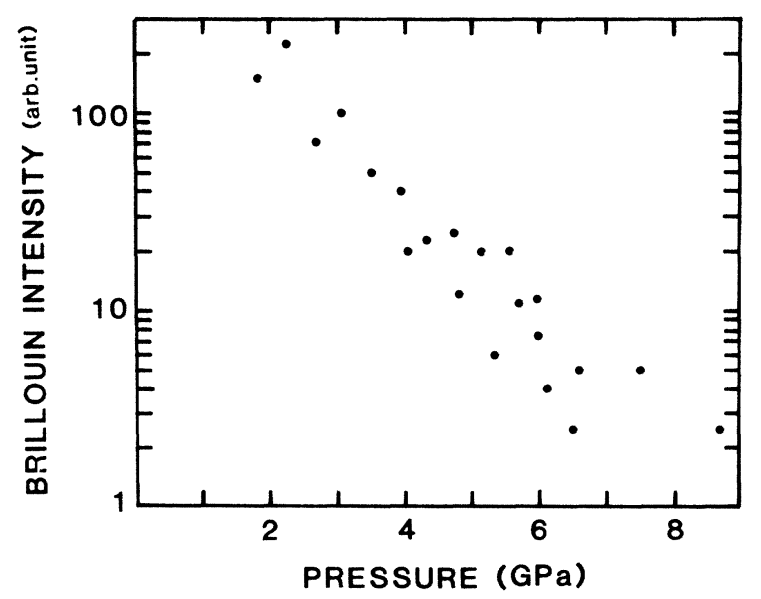

FIG. 7. Logarithm of the intensity of the Brillouin peak versus pressure. 


\section{THEORETICAL ANALYSIS}

The Brillouin scattering measurements reported in the preceding section and the recent $\mathrm{x}$-ray determination of the equation of state ${ }^{17-19}$ provide the first comprehensive data set of the evolution of the properties of a rare-gas solid at high densities. In the density range investigated here, electronic excitations are still negligible and the substance remains a simple closed-shell insulator. ${ }^{23}$ In the density region previously studied, solid argon was well modeled by pair potentials except at very low density near $P=0$, where three-body forces have to be incorporated. ${ }^{24}$ The pair potential is accurately determined in the region of the "well," while the repulsive part at small distances is not as precisely known. It is in this region that the existing potentials differ most markedly from one another and also from the experimental results obtained from beam scattering experiments. ${ }^{25}$ The experimental data now available in the high-density region provide an excellent opportunity for testing the various potentials presently used and should provide us with the following information: (i) Does the pair potential, as determined by beam scattering, explain the experimental data? and (ii) If not, can an effective pair potential (which included effects of three-body forces) be found which explains the data?

In a recent article ${ }^{26}$ on the high-pressure phase diagram of helium, the self-consistent harmonic (SCH) theory, corrected for the cubic anharmonic term near melting, was shown to be a reliable statistical model for analyzing properties of dense insulators. Since comprehensive reviews of the SCH method are available and since we have used the same program as in Ref. 23, here we will simply briefly review the basic ideas of the method.

In the SCH theory, the force constants used to calculate the dynamical matrix are self-consistently averaged over the position of the atoms undergoing the thermal motion. The structure is taken to be fcc, as determined by $x$ rays, ${ }^{27}$ which permits the sums to be carried out over $\frac{1}{48}$ of the total Brillouin zone with a mesh equivalent to 3999 points in the full zone. The frequencies and free energies converge to within two parts in $10^{4}$ after eight iterations of the self-consistent equations. Since in solid $\mathrm{He}$ the $\mathrm{SCH}$ free energy was found to be not accurate enough near melting and the first cubic anharmonic term $\Delta F_{3}$, to be required, we have also included it in these calculations. Since it involves a lengthy procedure with a double sum over the Brillouin zone, an estimate was obtained within the framework of an Einstein approximation, viz.,

$$
\begin{aligned}
\Delta F_{3}^{E}= & -\frac{A \hbar^{2}}{48 M^{3} \omega_{E}^{4}}\left[12 n\left(\omega_{E}\right)^{2}+12 n\left(\omega_{E}\right)+1\right] \\
& \times \sum_{l} \sum_{\alpha \beta \gamma} \Phi_{\alpha \beta \gamma}^{2}(l),
\end{aligned}
$$

where $\Phi_{\alpha \beta \gamma}$ is the average of the third derivative of the interaction potential over the Gaussian distribution of the displacements and

$$
n\left(\omega_{E}\right)=\left(e^{\beta \hbar \omega_{E}}-1\right)^{-1} \text {. }
$$

This approximation will be denoted by $\mathrm{SCH}$ plus $\mathrm{CE}$ (the cubic Einstein term). The constant $\boldsymbol{A}$ was introduced in order to compensate for the fact that by replacing all $\omega_{q \lambda}$ by the average value $\omega_{E}, \Delta F_{3}$ would be underestimated. In this paper we take $A=1.2614$, which was shown to result in a perfect agreement between $\mathrm{SCH}+\mathrm{CE}$ and Monte Carlo free energies in the near melting region for dense helium. ${ }^{28}$ This approximation should also be very accurate for argon since its potential in reduced units is similar to that of helium. Furthermore, this approximation represents a noticeable improvement over the $\mathrm{SCH}$ only for pressures less than $3 \mathrm{GPa}$ above the melting point; above this pressure the $\mathrm{SCH}$ is very accurate, even without the correction term. It has also been shown in Ref. 26 that the SCH dispersion curves of dense helium agree well with those calculated by molecular dynamics in the small- $k$ region, even near melting, implying that the isothermal elastic constants were well calculated in this approximation.

The calculations reported here were performed with four well-known potentials for argon. (i) The BarkerFisher-Watts (BFW) potential; fitted to pair, liquid, and solid properties and hence supposed to be accurate over a wide range of interatomic distances. ${ }^{29}$ (ii) The Ross potential; fitted to shock-wave data; expected to be good in the repulsive region. ${ }^{23}$ (iii) The HFD- $C$ potential of Aziz and Chen known as the best potential for argon, especially in the potential-well region..$^{30}$ (iv) HFD- $D$; a modification of HFD- $C$ that is forced into better agreement with beam

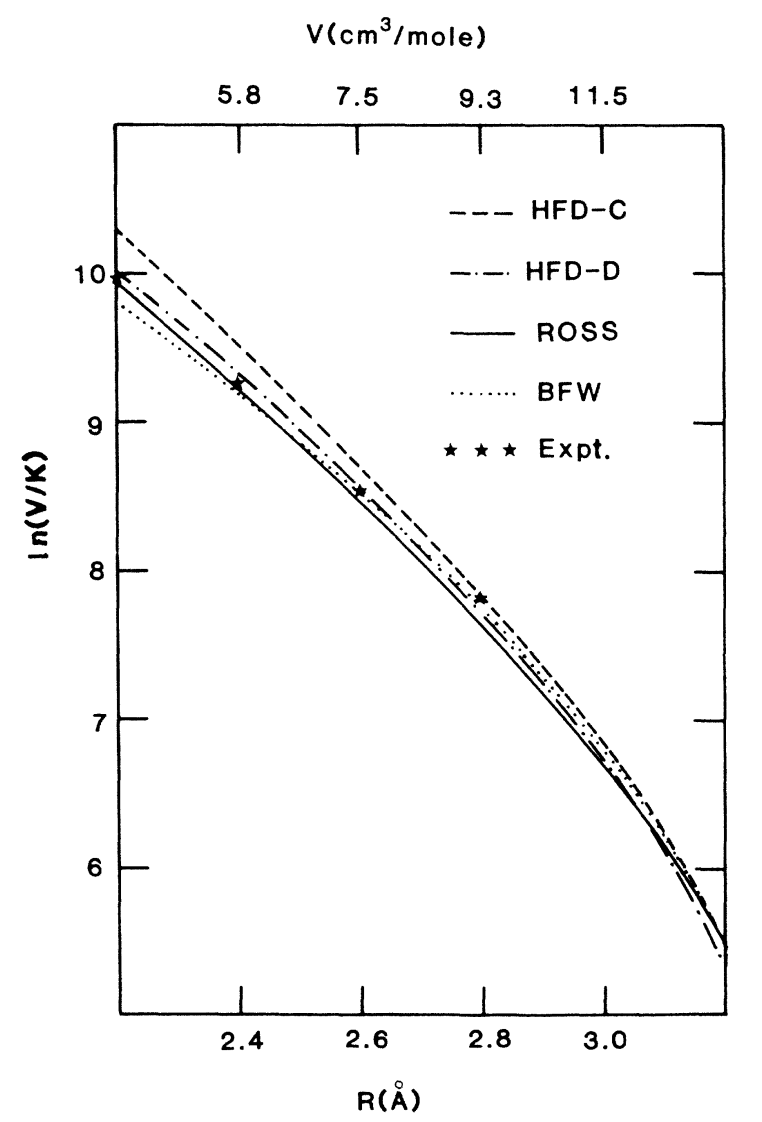

FIG. 8. Logarithm of the pair potentials of argon versus the interatomic distance. Each interatomic distance $R$ corresponds to the nearest-neighbor distance of a fcc lattice of volume $V=R^{3} / 2^{1 / 2}$. The potential labels are discussed in text. 
scattering data ${ }^{1}$ but which is less accurate in the well region. The repulsive parts of the above potentials, together with the beam scattering potential, are plotted in Fig. 8. The HFD- $C$ potential for $R \geqq 2.8 \AA$ and the beam scattering one for $R \leqq 2.8 \AA$ should be very near the true pair potential. Although many other potentials of argon have been proposed, we have not included them here since they fall within the range covered by the potentials we have chosen.

Since it was shown that both the equation of state and the elastic constants change by less than $5 \%$ when only first neighbors are included in the calculation, it can be inferred in the calculation that these properties are essentially determined by the shape of the potential around the nearest-neighbor distance (for a fcc crystal $R=2^{1 / 6} V^{1 / 3}$, where $V$ is the specific volume). With this in mind, in Fig. 8 we have also given a scale of specific volume that corresponds to a nearest-neighbor distance $\boldsymbol{R}$. In Figs. 9(a) and 9(b) we plot the equation of state, calculated us-
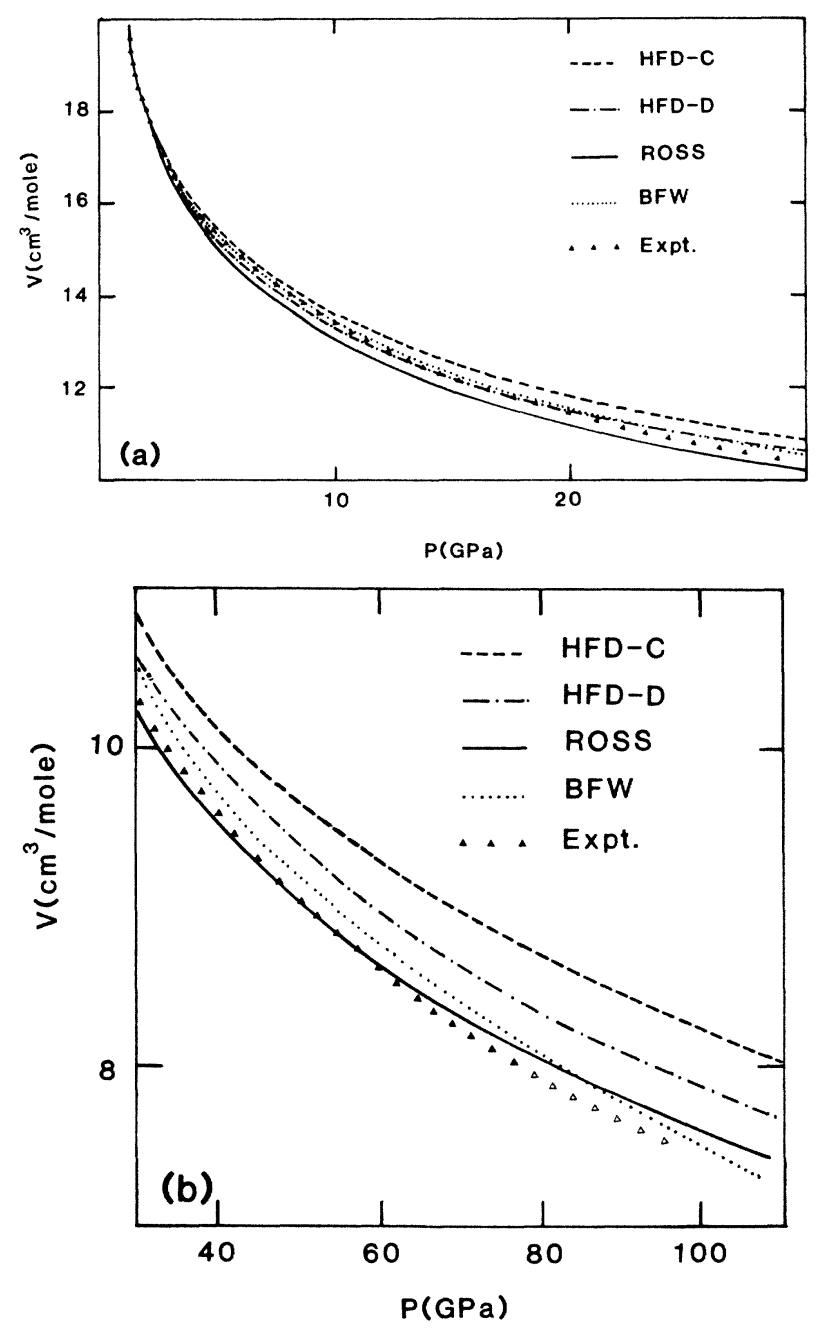

FIG. 9. (a) Equation of state of solid argon determined by the various potentials compared with the experimental one up to 30 $\mathrm{GPa}$. The experimental data represented by the fit of Eq. (6) are plotted as triangles. (b) Same as (a) in the high-pressure region: between 30 and $110 \mathrm{GPa}$. The open triangles represent extrapolation of the experimental EOS (Refs. 17-19) using Eq. (6). ing the potentials previously described, and compare it with that which was experimentally determined [for clarity, in this figure we have replaced the individual experimental points by the fit described by Eq. (6)]. The calculated elastic constant $C,{ }^{*}$ which can be directly compared with experiment, is presented in Fig. 10 as a function of pressure for the various potentials. The complete set of elastic constants calculated with the HFD- $C$ potential is given for a number of pressures in Table III, together with the experimental ones.

We point out that Fig. 9 it can be seen that above 0.7 $\mathrm{GPa}$, the properties that are being probed are determined by the potential in the region where substantial differences between the various models exist.

\section{COMPARISON OF THEORY AND EXPERIMENT}

In Fig. 9 it can be seen that below $3 \mathrm{GPa}$, essentially all the calculations are in agreement with experiment. This is expected since in this region the interatomic distances are still in a region where the pair potential is well known and where all the potentials used are similar. In the region between 3 and $10 \mathrm{GPa}(R=3.2 \AA)$, the BFW potential fits the experimental data extremely well, while the largest discrepancy is obtained with the Ross potential. For pressures between 10 and $30 \mathrm{GPa}(R \sim 3.0 \AA)$, no potential gives a good fit but the fits are slightly better for the potentials which are less repulsive and which have a smaller first derivative. The good agreement produced for the Ross potential around $35 \mathrm{GPa}$ is not surprising since the potential was made to fit the Hugoniot shock-wave data at $\sim 30 \mathrm{GPa}$ and happens to be the softer potential. At higher pressure ( $>60 \mathrm{GPa}$ ) all the potentials seem to deviate from the experimental results but the BFW becomes softer in this density region, its equation of state (EOS) initiates a better agreement.

The preceding analysis indicates that the best fit seems to be obtained with the less repulsive potentials which lie below the results obtained by beam scattering which are "perfect" two-body interactions. This implies that these potentials are effective two-body interactions which contain contributions arising from many-body forces. That

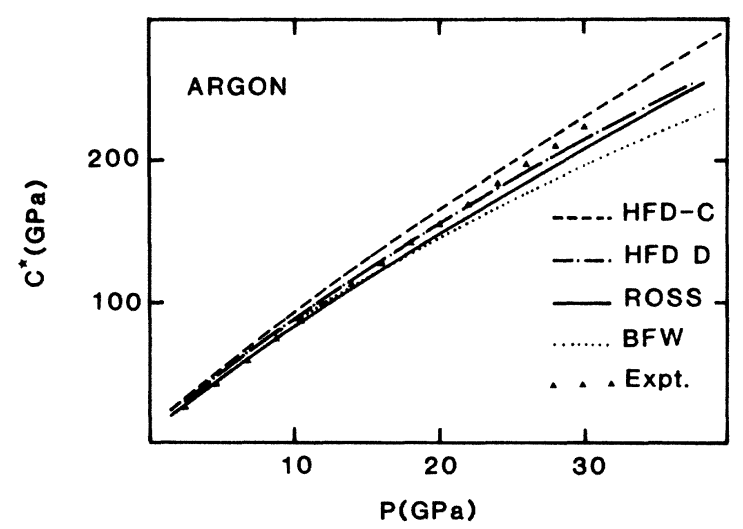

FIG. 10. Comparison between the elastic constant $C^{*}$ calculated for various potentials in the SHC formalism and our experimental results versus pressure. 
TABLE III. Experimental and theoretical HFD- $C$ (indicated by *) elastic constants. $\mathscr{A}=2 C_{44} /\left(C_{11}-C_{12}\right)$ is the anisotropy parameter and $\delta=\left(C_{44}-C_{12}+2 P\right) / C_{12}$ is the deviation from the Cauchy relation.

\begin{tabular}{llcccl}
\hline $\begin{array}{c}P \\
(\mathrm{GPa})\end{array}$ & $\begin{array}{c}C_{11} \\
(\mathrm{GPa})\end{array}$ & $\begin{array}{c}C_{12} \\
(\mathrm{GPa})\end{array}$ & $\begin{array}{c}C_{44} \\
(\mathrm{GPa})\end{array}$ & \multicolumn{1}{c}{} & \multicolumn{1}{c}{$\delta$} \\
\hline $1.65^{*}$ & 15.1 & 10.0 & 7.3 & 2.86 & 0.06 \\
2.0 & 16.0 & 8.4 & 7.5 & $>1.97$ & 0.37 \\
$3.21^{*}$ & 26.7 & 18.4 & 12.5 & 3.01 & 0.028 \\
4.0 & 32 & 16 & 12 & $>1.5$ & 0.25 \\
$5.8^{*}$ & 43.6 & 31.0 & 19.7 & 3.12 & 0.009 \\
6.0 & 48 & 22 & 18 & $>1.38$ & 0.36 \\
15 & 110 & 44 & 41 & $>1.24$ & 0.61 \\
20 & 140 & 54 & 54 & $>1.25$ & 0.74 \\
25 & 180 & 63 & 68 & $>1.16$ & 0.87 \\
30 & 210 & 74 & 80 & $>1.17$ & 0.89 \\
$30^{*}$ & 171 & 130 & 71 & 3.46 & 0.007 \\
$51^{*}$ & 268.3 & 209.5 & 108 & 3.67 & 0.002 \\
\hline \hline
\end{tabular}

this contribution is attractive in nature agrees with the results obtained from the study of rare-gas clusters. ${ }^{31}$ Our conclusion, however, differs from that in Ref. 23, in which the contribution of three-body forces was found to be negligible, but it must be stressed that the temperature domains in which the conclusions are drawn are not the same.

In a discussion of the elastic properties of Ar it is useful to consider the Cauchy relation which states that at zero temperature and pressure, in a crystal in which all atoms are at the center of symmetry and in which only central forces play a significant role, the relationship

$$
C_{44}=C_{12}
$$

must hold. ${ }^{22}$ At higher pressures this condition can be generalized $^{32}$ to

$$
C_{44}-C_{12}+2 P=0 \text {. }
$$

The effect of temperature on the Cauchy relationship can be estimated by considering anharmonic terms in the free energy and are found to be small (viz., $6 \%$ close to melting and $<1 \%$ above $6 \mathrm{GPa}$ ). Hence, as expected, the theoretical calculation (Table III) satisfies Eq. (17) to a good degree of accuracy, especially at high pressures. The experimental results in Table III, however, are inconsistent with Eq. (17). Even if one considers the upper bound for $C_{12}$ (viz., $C_{12}=B$ ), Eq. (17) is violated above
$15 \mathrm{GPa}$. Consequently, it can be stated that an accurate description of high-density argon in terms of pairwise interactions is impossible and that the incorporation of noncentral forces is absolutely necessary.

The above conclusion is confirmed by the calculation, as shown in Fig. 10, in which the elastic constant $C^{*}$ is plotted. As explained in Sec. III, the experimental $C^{*}$ is a lower bound. Even if one takes into account the errors due to the pressure determination, it can be seen that the potential which best fits the equation of state gives a $C^{*}$ out of the allowed range and provides the worst description of the elastic constants; or conversely, the change necessary to account for the elastic constant, viz., a hardening of the repulsive forces, produces a definitive deterioration of the equation of state.

\section{CONCLUSIONS}

The Brillouin scattering results presented in this article, together with previous $x$-ray measurements ${ }^{17-19}$ up to 77 $\mathrm{GPa}$, provide limit values for a complete set of elastic constants for argon up to $35 \mathrm{GPa}$. It is the first time that such a complete data set exists for any material at ultrahigh pressures and it has provided an ideal case for investigating the validity of the pair potential concept which is usually employed in calculations. Although reasonably accurate equations of state can be derived using potentials currently found in the literature, the elastic constants through the Cauchy relation indicate that a complete description of the elastic constants is not feasible using a pair potential model. The fact that noncentral forces must be incorporated to produce a complete description is also confirmed by the calculations and leads to the fact that many-body exchange potentials must be incorporated into any calculation which attempts to describe dense Ar and that there is no point in trying to refine an effective two-body potential.

\section{ACKNOWLEDGMENTS}

This work was supported by the Institut National d'Astronomie et de Géophysique under Grant No. 83070978 and the Commissariat á l'Energie Atomique under Grant No. 1617-A4. We wish also to thank J. M. Besson for a critical reading of the manuscript, D. Frenkel for communication of his results before publication, and $D$. Levesque and J. J. Weis for helpful discussions. Laboratoire de Physique des Milieux Très Condensés is Unité No. 782 associé au Centre National de la Recherche Scientifique.
*Permanent address: Materials Science and Technology Division, Argonne National Laboratory, 9700 South Cass Avenue, Argonne, IL 60439.

${ }^{1}$ R. A. Aziz, in Inert Gases, edited by M. L. Klein (SpringerVerlag, Berlin, 1984), p. 5.

${ }^{2}$ See, Rare Gas Solids, edited by J. A. Venables and M. L. Klein (Academic, New York, 1976), Vols. I and II.
${ }^{3}$ A. Jayaraman, Rev. Mod. Phys. 55, 65 (1983).

${ }^{4}$ C. H. Whitfield, E. M. Brody, and W. A. Bassett, Rev. Sci. Instrum. 47, 942 (1976).

${ }^{5}$ H. Shimizu, E. M. Brody, H. K. Mao, and P. M. Bell, Phys. Rev. Lett. 47, 128 (1981).

${ }^{6}$ A. Polian, J. M. Besson, M. Grimsditch, and H. Vogt, Phys. Rev. B 25, 2767 (1982). 
${ }^{7}$ A. Polian and M. Grimsditch, Phys. Rev. Lett. 52, 1312 (1984).

${ }^{8}$ G. J. Piermarini, S. Block, J. D. Barnett, and R. A. Forman, J. Appl. Phys. 46, 2774 (1975).

${ }^{9}$ See, for instance, H. Z. Cummins and P. E. Schoen, in Laser Handbook, edited by F. T. Arecchi and E. O. Schulz-Dubois (North-Holland, Amsterdam, 1972), p. 1029.

${ }^{10}$ M. H. Grimsditch and A. K. Ramdas, Phys. Rev. B 11, 3139 (1975).

${ }^{11}$ A. Polian and M. Grimsditch, Phys. Rev. B 27, 6409 (1983).

${ }^{12}$ H. K. Mao, P. M. Bell, J. W. Shaner, and D. J. Steinberg, J. Appl. Phys. 49, 3276 (1978).

${ }^{13}$ D. H. Liebenberg, R. L. Mills, and J. C. Bronson, J. Appl. Phys. 45, 741 (1974).

${ }^{14} \mathrm{~J}$. P. Itie, thesis, Universite de Paris, 1984, and J. P. Itie and R. Letoullec, J. Phys. (Paris) Colloq. 45, C8-53 (1984).

${ }^{15}$ M. Grimsditch, R. Letoullec, A. Polian, and M. Gauthier (unpublished).

${ }^{16}$ M. Lallemand and D. Vidal, J. Chem. Phys. 66, 4776 (1977).

${ }^{17}$ R. M. Hazen, H. K. Mao, L. W. Finger, and P. M. Bell; Carnegie Inst. Washington Yearb. 79, 348 (1980).

${ }^{18} \mathrm{G}$. Zou, H. K. Mao, and P. M. Bell, Carnegie Inst.
Washington Yearb. 81, 392 (1982).

${ }^{19}$ J. Xu, H. K. Mao, and P. M. Bell, High Temp. High Pressures 16, 495 (1984).

${ }^{20}$ See, B. P. Stoicheff, in Ref. 2, p. 979.

${ }^{21}$ P. M. Bell and H. K. Mao, Carnegie Inst. Washington Yearb. 80, 404 (1981).

22See, Dynamical Theory of Crystal Lattices, edited by M. Born and K. Huang (Clarendon, Oxford, 1954), p. 373.

${ }^{23}$ M. Ross, J. Chem. Phys. 73, 4445 (1980).

${ }^{24}$ R. Aziz, J. Chem. Phys. 72, 5787 (1980).

${ }^{25}$ See, Ref. 1, p. 80.

26P. Loubeyre, D. Levesque, and J. J. Weis, Phys. Rev. B 33, 318 (1986).

${ }^{27}$ F. Simon and C. von Simson, Z. Phys. 25, 160 (1924).

${ }^{28} \mathrm{D}$. Frenkel (private communication).

${ }^{29}$ J. A. Barker, R. A. Fischer, and R. O. Watts, Mol. Phys. 21, 657 (1971).

${ }^{30}$ R. A. Aziz and H. H. Chen, J. Chem. Phys. 67, 5719 (1977).

${ }^{31}$ E. E. Polymeropoulos, P. O. Bopp, J. Brickmann, L. Jansen, and R. Block, Phys. Rev. A 31, 3565 (1985).

32P. Korpiun and E. Lüscher, Ref. 2, p. 729. 